\title{
Adult Neurogenesis Conserves Hippocampal Memory Capacity
}

\author{
(D)Md Jahangir Alam, ${ }^{1,2}$ Takashi Kitamura, ${ }^{4}$ Yoshito Saitoh, ${ }^{1,2}$ Noriaki Ohkawa, ${ }^{1,2}$ Takashi Kondo, ${ }^{3}$ \\ and $\odot_{\text {Kaoru Inokuchi }}{ }^{1,2}$ \\ ${ }^{1}$ Department of Biochemistry, Graduate School of Medicine and Pharmaceutical Sciences, ${ }^{2}$ Core Research for Evolutional Science and Technology, Japan \\ Science and Technology Agency, and ${ }^{3}$ Department of Radiological Sciences, Graduate School of Medicine and Pharmaceutical Sciences, University of \\ Toyama, Toyama 930-0194, Japan, and ${ }^{4}$ Department of Psychiatry, Department of Neuroscience, University of Texas Southwestern Medical Center, Dallas, \\ Texas 75390
}

The hippocampus is crucial for declarative memories in humans and encodes episodic and spatial memories in animals. Memory coding strengthens synaptic efficacy via an LTP-like mechanism. Given that animals store memories of everyday experiences, the hippocampal circuit must have a mechanism that prevents saturation of overall synaptic weight for the preservation of learning capacity. LTD works to balance plasticity and prevent saturation. In addition, adult neurogenesis in the hippocampus is proposed to be involved in the downscaling of synaptic efficacy. Here, we show that adult neurogenesis in male rats plays a crucial role in the maintenance of hippocampal capacity for memory (learning and/or memory formation). Neurogenesis regulated the maintenance of LTP, with decreases and increases in neurogenesis prolonging or shortening LTP persistence, respectively. Artificial saturation of hippocampal LTP impaired memory capacity in contextual fear conditioning, which completely recovered after $14 \mathrm{~d}$, which was the time required for LTP to decay to the basal level. Memory capacity gradually recovered in parallel with neurogenesis-mediated gradual decay of LTP. Ablation of neurogenesis by $\mathrm{x}$-ray irradiation delayed the recovery of memory capacity, whereas enhancement of neurogenesis using a running wheel sped up recovery. Therefore, one benefit of ongoing adult neurogenesis is the maintenance of hippocampal memory capacity through homeostatic renewing of hippocampal memory circuits. Decreased neurogenesis in aged animals may be responsible for the decline in cognitive function with age.

Key words: adult neurogenesis; hippocampus; learning and memory; memory capacity; synaptic plasticity

\section{Significance Statement}

Learning many events each day increases synaptic efficacy via LTP, which can prevent the storage of new memories in the hippocampal circuit. In this study, we demonstrate that hippocampal capacity for the storage of new memories is maintained by ongoing adult neurogenesis through homoeostatic renewing of hippocampal circuits in rats. A decrease or an increase in neurogenesis, respectively, delayed or sped up the recovery of memory capacity, suggesting that hippocampal adult neurogenesis plays a critical role in reducing LTP saturation and keeps the gate open for new memories by clearing out the old memories from the hippocampal memory circuit.

\section{Introduction}

The hippocampus is crucial for declarative memories in humans (Scoville and Milner, 1957; Ofen et al., 2007) and encodes epi-

Received Oct. 15, 2017; revised April 19, 2018; accepted May 12, 2018.

Author contributions: M.J.A., T. Kitamura, and K.I. designed research; M.J.A., T. Kitamura, Y.S., N.O., and T. Kondo performed research; M.J.A. analyzed data; M.J.A., T. Kitamura, and K.I. wrote the paper.

This work was supported by the Core Research for Evolutional Science and Technology (CREST) program (JPMJCR13W1) of the Ministry of Education, Culture, Sports, Science and Technology (MEXT) Science and Technology Agency, Japan Society for the Promotion of Science (JSPS KAKENHI Grant JP23220009), MEXT (Grant-in-Aid for Scientific Research on Innovative Areas "Memory Dynamism" Grant JP25115002, the Mitsubishi Foundation, the Uehara Memorial Foundation, and the Takeda Science Foundation (K.I.). T. Kitamura was funded by Sasagawa Scientific Grants (22-440). We thank Nakamura-Sekizenkai (K2012011006) for supporting M.J.A., K. Choko for sodic and spatial memories in animals (Conway, 2009; Buzsáki and Moser, 2013). During memory formation, changes in synaptic strength, LTP, and LTD at appropriate synapses are both necessary and sufficient for information storage (Nabavi et al., 2014).

setting up the LTP experiments, and M. Shehata and M. Nomoto for valuable discussions on behavioral analysis and the manuscript.

The authors declare no competing financial interests.

Correspondence should be addressed to Kaoru Inokuchi, Department of Biochemistry, Faculty of Medicine, Graduate School of Medicine and Pharmaceutical Sciences, University of Toyama, 2630 Sugitani, Toyama 930-0194 Japan. E-mail: inokuchi@med.u-toyama.ac.jp.

DOl:10.1523/JNEUROSCI.2976-17.2018

Copyright $\odot 2018$ the authors $\quad 0270-6474 / 18 / 386854-10 \$ 15.00 / 0$ 
Hippocampus-dependent learning basically induces strengthening of synaptic efficacy through potentiation of synapses in the hippocampus (Hebb, 1949; Whitlock et al., 2006; Tononi and Cirelli, 2014). This implies that information coding prevents the storing of new information in the neuronal circuits. Under physiological conditions, a balance between LTP and LTD is maintained to avoid saturation of the neuronal network (Collingridge et al., 2010). In addition, sleep plays an important role in memory formation by removing and down-sizing spines and thereby scaling down excitatory synapses (Tononi and Cirelli, 2014; Li et al., 2017; de Vivo et al., 2017) to avoid saturation of the circuits.

Throughout adulthood, new neurons are continuously generated and functionally integrated into existing circuits in the hippocampal dentate gyrus (DG) (Altman and Das, 1965; Zhao et al., 2008; Deng et al., 2010; Kitamura and Inokuchi, 2014). Inhibition or enhancement of hippocampal neurogenesis, respectively, prolongs or shortens the hippocampus-dependent period of memory, indicating that it is involved in the modulation of the hippocampus-dependent period of contextual fear memory (Kitamura et al., 2009; Kitamura and Inokuchi, 2014). Moreover, decreased neurogenesis facilitates the long-lasting maintenance of DG LTP (Kitamura et al., 2009). In the present study, we show that adult neurogenesis plays an important role in the maintenance of hippocampal memory capacity and enables animals to learn new events through the renewal of hippocampal memory circuit.

\section{Materials and Methods}

Animals. The Animal Care and Use Committee of the University of Toyama, in compliance with the guidelines of the National Institutes of Health, approved the use and care of animals in this study. Five-week-old [for the repeated maximum electroconvulsive shock (rMECS) experiments] and 8-week-old [for the repeated high-frequency tetanic stimulation (rHFS) experiments] Wister ST male rats were purchased from Sankyo Laboratory. Food and water were provided ad libitum. All animals were maintained at room temperature and a 12:12 h light/dark cycle (8:00 A.M. to 8:00 P.M.).

Ibotenic acid (IBO) injection. For the LTP saturation experiments (see Figs. 2, 3, 4) in freely moving animals, all rats in the control and experimental groups, except those used in Figure $3, I-K$, underwent complete unilateral hippocampal (left hemisphere) lesions by IBO (CAS 255255-8; Santa Cruz Biotechnology) injection at multiple sites (Jarrard, 1989). The IBO was dissolved in PBS at a concentration of $10 \mathrm{mg} \mathrm{ml}^{-1}$ and a $\mathrm{pH}$ of 7.4. The dissolved IBO solution was divided into aliquots and stored in a $-30^{\circ} \mathrm{C}$ freezer. On the day of injection, an IBO aliquot was thawed at room temperature and used for a maximum of $2 \mathrm{~d}$, with any remaining solution then being discarded. During the surgery, rats were anesthetized with an intraperitoneal injection (i.p) of sodium pentobarbital ( $65 \mathrm{mg} \mathrm{kg}^{-1}$ of body weight). To achieve a complete lesion of the left hippocampus, IBO was injected into multiple sites (14 sites; see Table 1) using a $2 \mu$ l Hamilton syringe and a guide cannula (EIM-362; Eicom). The quantity of IBO injected at each site varied from 0.05 to $0.1 \mu \mathrm{l}$. The

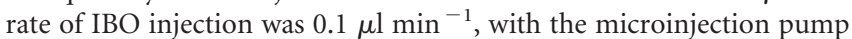
pressure set at $30 \%$ (Legato 111; KD Scientific). The guide cannula was kept in place for an additional minute to prevent spreading. After completion of injections, wounds were closed with dental cement, the animals were placed in their home cages (HC), and their health condition was monitored until recovery from anesthesia was complete. After completing the full course of experiments, post hoc analyses were performed in all animals to check that the lesion to the left side of the hippocampus was complete by staining with cresyl violet.

Saturation of hippocampal LTP in freely moving rats. Ten days after the IBO injection into the left hippocampus, a second surgery was conducted to implant electrodes into the right hippocampus. A bipolar stimulating electrode was positioned in the perforant pathway (PP) to selectively stimulate the PP and projections and a monopolar recording electrode was placed in the DG. Tungsten wire was used to make the electrodes. To stimulate the medial perforant pathway (Matsuo et al., 2000; Fukazawa et al., 2003), the stimulating electrode was placed $7.8 \pm 0.3 \mathrm{~mm}$ posterior, $4.8 \pm 0.3 \mathrm{~mm}$ lateral, and $4.2 \pm 0.3 \mathrm{~mm}$ inferior to the bregma. The recording electrode was implanted ipsilaterally $4.0 \pm 0.2 \mathrm{~mm}$ posterior, $2.5 \pm 0.3 \mathrm{~mm}$ lateral, and $3.5 \pm 0.3 \mathrm{~mm}$ inferior to the bregma. Rats were permitted to recover for at least $10 \mathrm{~d}$ in individual HCs. Throughout the recovery time, input/output curves were determined as a function of current intensity $(0.1-1.0 \mathrm{~mA})$. A current that evoked $50 \%$ of the maximum fEPSP slope amplitude was used as a threshold for all rHFS and test pulse experiments and then the value was kept constant throughout the entire experiment. Test stimuli were delivered at $20 \mathrm{~s}$ intervals to record the fEPSP. LTP was induced by delivering HFS as described previously, with slight modifications (Matsuo et al., 2000; Fukazawa et al., 2003). The HFS (500) was used to stimulate the PP and projections, with each HFS (500) containing 10 trains with 1 min intertrain intervals. Each train consisted of 5 bursts of 10 pulses at $400 \mathrm{~Hz}$ delivered at $1 \mathrm{~s}$ interburst intervals. After baseline recording for $15 \mathrm{~min}$, LTP was induced by delivering HFS stimulation. Each animal received two HFS stimulation sessions per day with a $1.5 \mathrm{~h}$ interval between sessions. All animals (except for those in the test pulse group) received a total of 6 HFS stimulation sessions in $3 \mathrm{~d}$. The seventh HFS stimulation was given on the fourth day to check whether the hippocampal synaptic plasticity was saturated or if there would be further cumulative potentiation. Synaptic responses from the DG were measured for 15 min after each HFS stimulation session. The positions of the electrodes were analyzed after the experiments. Synaptic responses disappeared during the recovery period after surgery and/or in the middle of the rHFS stimulation in some animals regardless of the group. These animals were excluded from the study.

rMECS stimulation. The rats received a series of 10 rMECS, 1 every $2 \mathrm{~d}$, for $20 \mathrm{~d}$ (Stewart et al., 1994) under isoflurane anesthesia (2\% isoflurane with $2 \% \mathrm{O}_{2}$ ). Note that the animals in the rMECS study did not receive ibotenic acid injections. MECS $(100 \mathrm{~Hz}, 0.5 \mathrm{~ms}, 1 \mathrm{~s}, 65 \mathrm{~mA})$ was delivered by a pulse generator (57800-001; UGO BASILE) and the electrodes were attached to the ears via ear clips. After delivering the shock, the ear clip was left in place for an additional $10 \mathrm{~s}$ before being removed. These parameters were sufficient to induce seizures in all animals. The sham group received the relevant procedures except for the shock stimulation.

DG-LTP in urethane-anesthetized animals. After the behavioral (contextual fear conditioning; CFC) analysis, rMECS-treated or sham rats were used for the analysis of DG-LTP under urethane anesthesia (Inokuchi et al., 1996), as shown in Figure 5, D and E. Rats were anesthetized using urethane (i.p., $1.0 \mathrm{~g} \mathrm{~kg}^{-1}$ body weight), and the stimulating and recording electrodes were implanted unilaterally, in the medial perforant pathway (MPP) and DG, respectively (coordinates, recording electrode: AP: $4.0 \pm 0.2$, ML: $2.5 \pm 0.3, \mathrm{Z}: 3.5 \pm 0.3$, stimulating electrode: AP: $7.8 \pm 0.3$, ML: $4.5 \pm 0.3, \mathrm{Z}: 4.2 \pm 0.3)$. All the stimuli were conducted using biphasic square wave pulses ( $200 \mu$ s width) with the current intensity set to the level that evoked $50 \%$ of the maximum fEPSP slope amplitude. After monitoring a stable basal transmission for at least $15 \mathrm{~min}$, LTP was induced by delivering 100 pulses at $400 \mathrm{~Hz}$, hereafter referred to as HFS (100). HFS (100) contained two trains with 1 min intertrain intervals. Each train consisted of 5 bursts of 10 pulses at $400 \mathrm{~Hz}$, delivered at $1 \mathrm{~s}$ interburst intervals. The fEPSP slope was monitored by delivering test stimuli at $20 \mathrm{~s}$ intervals. A small animal heat controller (ATC-101B; Unique Medical, Japan) was used to maintain the body temperature of the animals at $37^{\circ} \mathrm{C}$ throughout the LTP experiments, which were performed under urethane anesthesia.

$X$-ray irradiation. Either 5-week-old rats or IBO-injected rats of $\sim 9-11$ weeks of age were irradiated. All animals were anesthetized using pentobarbital (i.p. $35 \mathrm{mg} \mathrm{kg}^{-1}$ of body weight) before being irradiated. The completely anesthetized animals were placed inside the $\mathrm{x}$-ray irradiation apparatus (MBR-1520R-3, Hitachi, Japan) and irradiated at 150 $\mathrm{kV}$ and $5 \mathrm{~mA}$. For irradiation, $0.5 \mathrm{~mm}$ aluminum and $0.2 \mathrm{~mm}$ copper filters were used. The animal's entire body was covered with a lead shield to protect it from the $\mathrm{x}$-ray exposure, while the head was left exposed to the irradiation. The distance between the animal's head skin surface to the irradiation source was $23.3 \mathrm{~mm}$. The procedure lasted for $10 \mathrm{~min}$, 


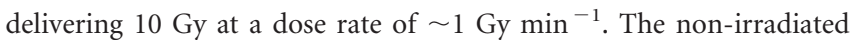
( $0 \mathrm{~Gy}$ ) group received all the processes except for the irradiation.

Running wheel $(R W)$ experiment. Six-week-old rats were placed in cages equipped with a RW for a period of 6 weeks. Cycle computers were attached to the cages with the sensors placed on the back of the wheel to analyze usage. One day after x-ray irradiation, rats were placed inside the cages equipped with a RW. The nonrunner group was kept in the HC. Two weeks later, IBO injection and electrode implantation were performed in the left and right hemispheres of the brain. The rats were allowed a $5 \mathrm{~d}$ recovery period in the $\mathrm{HC}$, before being returned to the RW cages for the rest of the experiment.

$C F C$. All the rats were maintained in individual HCs with laboratory bedding. Training and testing sessions were conducted during the daytime in a soundproof behavioral room, which was positioned adjacent to the animal living room. The behavioral experiments were performed as described previously (Kitamura et al., 2009). The conditioning chamber consisted of Plexiglas front and black sides and back walls (width $\times$ depth $\times$ height: $175 \times 165 \times 300 \mathrm{~mm}$ ). The chamber floor consisted of 21 stainless steel rods with a diameter of $2 \mathrm{~mm}$ placed $4 \mathrm{~mm}$ apart. A shock generator was connected to the rods via a cable harness. During the training phase, rats were placed in the conditioning chamber and, after 3 min, received a single foot shock for $2 \mathrm{~s}$ ( $1.3 \mathrm{~mA}$ for HFS-treated animals, Figs. 1, 2, 3, 4; and 1.6 mA for rMECS-treated animals, Fig. 5). After the shock, animals remained in the chamber for an additional 1 min before being returned to their HCs. After $1 \mathrm{~d}$, the rats were placed back into the conditioning chamber for $4 \mathrm{~min}$ for a test session. After the end of each session, rats were returned to their HCs and the floor of the chamber was cleaned with distilled water and $70 \%$ ethanol. Scoring of the freezing response was conducted using an automated video tracking system $(\mathrm{Mu}-$ romachi Kikai). A freezing score was counted after $3.0 \mathrm{~s}$ of sustained freezing.

Auditory fear conditioning (AFC). AFC was used as a hippocampusindependent learning task. The conditioning chamber was similar to that used in the CFC experiments. During the training session, rats were placed in the conditioning chamber for $3 \mathrm{~min}$. After $2 \mathrm{~min}$, rats received a tone $(85 \mathrm{~dB}, 1 \mathrm{kHz}, 20 \mathrm{~s})$ co-terminated with a foot shock (the same as in the CFC task). After the shock, rats remained in the chamber for an additional $40 \mathrm{~s}$ before being returned to their HCs. After $1 \mathrm{~d}$, the rats were placed in a different chamber (triangular in shape) for the test. The test session lasted for $4 \mathrm{~min}$, with a $2 \mathrm{~min}$ pre-tone session followed by a continuous tone being delivered for $2 \mathrm{~min}$. After the end of each session, the rats were returned to their HCs and the chamber's floor was cleaned with distilled water and 70\% ethanol. Freezing response was measured using the automated video-tracking system.

Motility calculation. The motility of the rats during the CFC was measured using the previously mentioned video-tracking system (Kitamura et al., 2009, 2012). Motility was calculated for the first $3 \mathrm{~min}$ of the conditioning session (before receiving the shock) as the area covered by the rat's movement.

Cresyl violet staining. The procedure for cresyl violet (CAS 10510-54-0; Sigma-Aldrich) staining was similar to that described previously (Deitch and Moses, 1957; Humason, 1983). Animals were deeply anesthetized using an overdose injection of pentobarbital and then perfused transcardially using cold PBS, pH 7.4, followed by $4 \%$ paraformaldehyde in PBS. The brains were removed and further postfixed in $4 \%$ paraformaldehyde in PBS at $4^{\circ} \mathrm{C}$ for at least $72 \mathrm{~h}$. Horizontal sections $(20 \mu \mathrm{m})$ were cut on a cryostat and 5 sections were collected from the hippocampus starting at $3.80 \mathrm{~mm}$ bregma and ending at $6.0 \mathrm{~mm}$ bregma for staining. Brain sections were inoculated for $12-14 \mathrm{~min}$ in the cresyl violet solution, which was kept in an oven $\left(60^{\circ} \mathrm{C}\right)$. After washing the sections with distilled water, they were dehydrated with $70 \%$ alcohol, $95 \%$ alcohol, $100 \%$ alcohol, and then xylene. A similar pattern of hippocampal lesions was observed in all IBO-injected animals. No animals were excluded from the analyses.

BrdU and NeuN labeling and quantification. Procedures for the quantification of hippocampal cell proliferation and neurogenesis followed those described previously (Kitamura et al., 2009; Ohkawa et al., 2012). For counting of cell proliferation, rats received a single injection of BrdU (100 $\mathrm{mg} \mathrm{kg}^{-1}$, i.p.; Sigma-Aldrich; BrdU dissolved in $0.9 \% \mathrm{NaCl}$ solu-
Table 1. Coordinates for IBO injection

\begin{tabular}{lll}
\hline Coordinates (in mm) & & \\
\hline A-P & M-L & D-V \\
\hline 2.2 & 0.8 & 4.2 \\
3.0 & 1.5 & $3.6^{*}, 4.4^{*}$ \\
3.0 & 3.0 & 4.0 \\
4.0 & 2.5 & $3.5^{*}, 4.5^{*}$ \\
4.0 & 3.6 & 4.0 \\
4.8 & 3.2 & 4.5 \\
4.8 & 4.3 & $4.2,6.0$ \\
5.7 & 4.1 & 4.5 \\
5.7 & 5.1 & $4.0^{*}, 4.8,5.6$ \\
\hline
\end{tabular}

The bregma was used as a zero point to measure the anterior to posterior $(A-P)$ and medial to lateral $(M-L)$ coordinates. Dorsal to ventral ( $D-V)$ positions were measured from the skull at the bregma. Bregma and lambda were on the same horizontal plane. In some A-P coordinates, multiple $M-L$ and $D-V$ positions were used to inject the IBO. The injection quantity of the IBO for each position was $0.1 \mu$, except for the star $\left(^{*}\right)$ denoted mark, where $0.05 \mu$ l was injected.

tion); $2 \mathrm{~h}$ after the BrdU injection, they were anesthetized using an overdose of pentobarbital solution and transcardially perfused with cold PBS followed by $4 \%$ paraformaldehyde in PBS. For analysis of neurogenesis in the rHFS and test pulse groups, rats received $3 \mathrm{BrdU}$ injections ( 1 per day for 3 successive days) and were perfused $1 \mathrm{~d}$ after the CFC experiment. For analysis of neurogenesis in the rMECS and sham groups, rats received $6 \mathrm{BrdU}$ injections ( 2 per day for 3 consecutive days) and were perfused $1 \mathrm{~d}$ after the CFC experiments. After the rats were perfused with paraformaldehyde, the brains were removed and further postfixed in $4 \%$ paraformaldehyde in PBS for $24 \mathrm{~h}$ at $4^{\circ} \mathrm{C}$. The brains were then equilibrated overnight in 25\% sucrose in PBS before being frozen in dry ice powder. For BrdU staining, $20 \mu \mathrm{m}$ coronal sections were cut on a cryostat, with 1 in ever 3 sections from the hippocampus being collected (3.0-5.0 $\mathrm{mm}$ from the bregma; 30 sections from each rat). The brain sections were boiled in $0.01 \mathrm{~m}$ sodium citrate buffer, $\mathrm{pH}$ 6.0, for $10 \mathrm{~min}$ and then treated with $2 \mathrm{M} \mathrm{HCl}$ for $30 \mathrm{~min}$. The sections were then rinsed with $0.1 \mathrm{~m}$ boric acid, $\mathrm{pH} 8.5$, for $10 \mathrm{~min}$. Blocking of the sections was performed using blocking solution (5\% donkey serum in PBS containing $0.1 \%$ Triton X-100) at room temperature for $1 \mathrm{~h}$. After blocking, sections were incubated with blocking solution containing rat anti-BrdU (1:800, OBT0030, Bio-Rad/AbD Serotec; RRID:AB_609568) and mouse antiNeuN (1:300, MAB377, Millipore; RRID:AB_2298772) antibodies. After washing with $\mathrm{PBS}$, the sections were incubated with anti-rat IgG-Alexa Fluor 488 (1:200, A-21208, Invitrogen; RRID:AB_141709) and antirabbit IgG-Alexa Fluor 546 (1:200, A10036, Thermo Fisher Scientific; RRID:AB_2534012) antibodies at room temperature for $3 \mathrm{~h}$. The slides were then washed three times with PBS (10 min per wash). Sections were mounted on glass slides with Pro-Long Gold antifade reagents (Invitrogen). Hippocampal fluorescent images were obtained on a Keyence microscope (BZ-9000). After the images were acquired, the numbers of $\mathrm{BrdU}^{+}$and $\mathrm{BrdU}^{+} / \mathrm{NeuN}^{+}$cells were counted manually from 30 sections per rat using software supplied with the Keyence microscope.

Statistics. All data are presented as mean \pm SEM. Statistical analyses were performed using Prism version 6 software (GraphPad). The number of animals is indicated by " $n$." Comparisons between the data of two groups were analyzed using unpaired Student's $t$ tests or paired $t$ tests (within the same group). If the data did not meet the assumptions of the $t$ test, they were analyzed using the nonparametric Mann-Whitney $U$ test. Multiple group comparisons were assessed using one-way, two-way, or repeated-measures ANOVA, followed by the appropriate post hoc test when significant main effects or interactions were detected. The null hypothesis was rejected at the $p<0.05$ level.

\section{Results}

Recovery of hippocampal memory capacity from the saturation level correlates with LTP decay

DG plays an important role in both fear acquisition and retrieval during the CFC task (Liu et al., 2012; Bernier et al., 2017). CFC is a useful behavioral paradigm for analyzing hippocampal function because the hippocampus plays a critical role in acquiring 

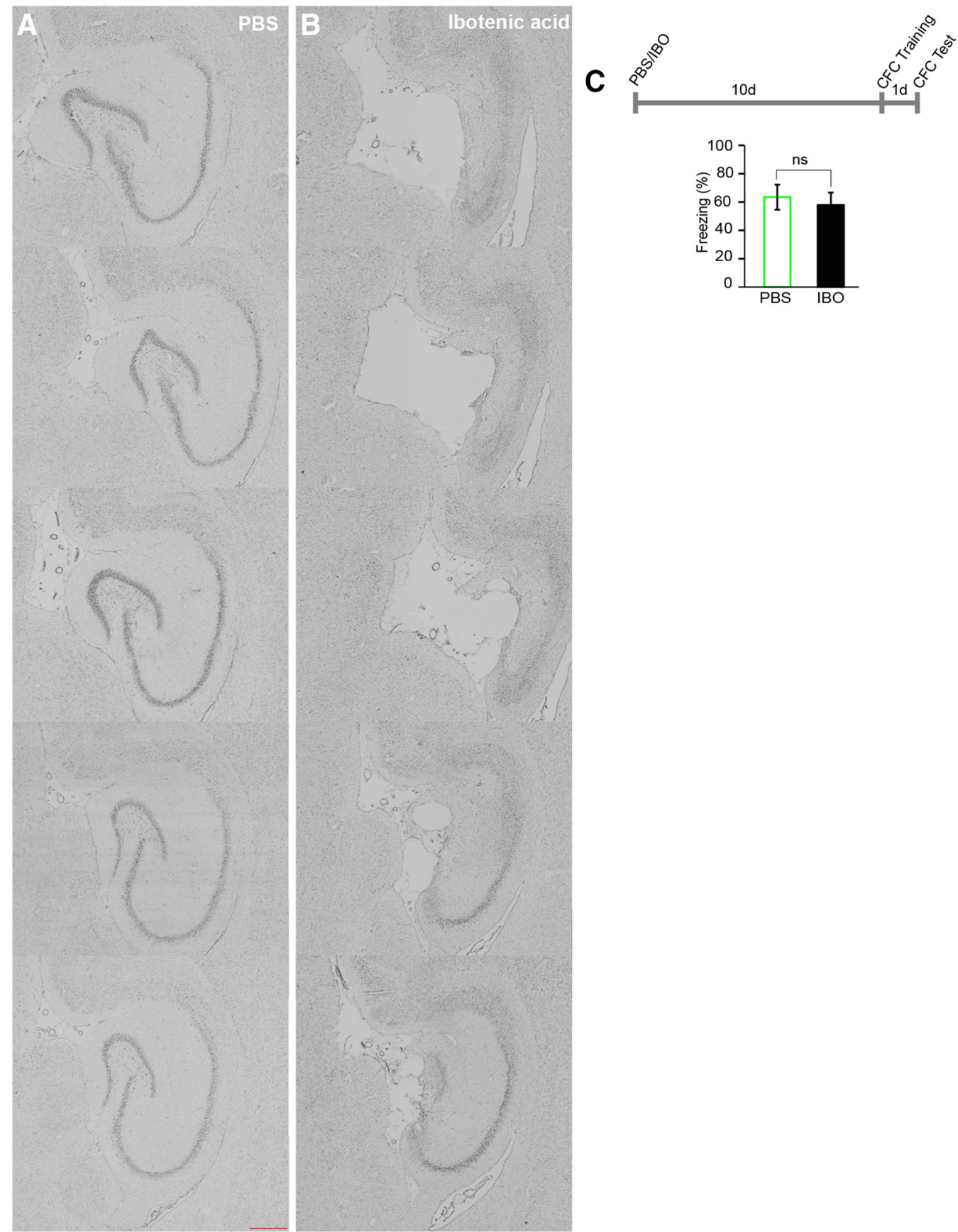

Figure 1. Unilateral IBO injection had no effect on hippocampal learning. $A, B$, Images showing the sequential horizontal hippocampal sections from the dorsal to the ventral part of the hippocampus from PBS-injected $(\boldsymbol{A})$ and IBO-injected $(\boldsymbol{B})$ rats after cresyl violet staining. Scale bar, $200 \mu \mathrm{m}$. $\boldsymbol{C}$, Experimental schedule and average freezing response observed in PBS- and IBO-injected rats during test sessions for the CFC task (unpaired $t$ test, $p=0.66, t_{9}=0.44 ; \mathrm{IBO}, n=6 ; \mathrm{PBS}, n=5$ rats).

and retrieving neural representations of the context in which fear becomes conditioned (Fanselow, 2010).

Two experimental approaches were used to saturate hippocampal synaptic efficacy in rats: rHFS (Castro et al., 1989; Moser et al., 1998) and rMECS (Stewart et al., 1994). In the first series of experiments, hippocampal synaptic efficacy was saturated by delivering rHFS to the $\mathrm{PP}$ of the right hemisphere, which projects to the DG of the dorsal hippocampus. To increase the sensitivity of animals to saturation of plasticity at synapses that might be used for learning (Moser et al., 1998), we damaged the dorsal hippocampus of the left hemisphere by injecting IBO at multiple sites (Jarrard, 1989) (Table 1). Cresyl violet staining showed that the multiple IBO injections completely damaged the dorsal hippocampus (Fig. 1A,B). Unilateral IBO injections had no effect on hippocampus-dependent memory (CFC) compared with injection of the vehicle (PBS) control when tested $1 \mathrm{~d}$ after the training (Fig. 1C).

After a $10 \mathrm{~d}$ recovery period from IBO injection, stimulating and recording electrodes were implanted into the right hemisphere (Fig. $2 A, B$ ). rHFS was delivered to the PP-DG pathway and the evoked synaptic responses were measured from the DG in freely moving, nonanesthetized rats. The application of rHFS gradually increased the slope of the fEPSP, which reached saturation level after the fifth stimulation session (Fig. 2C,D). One 

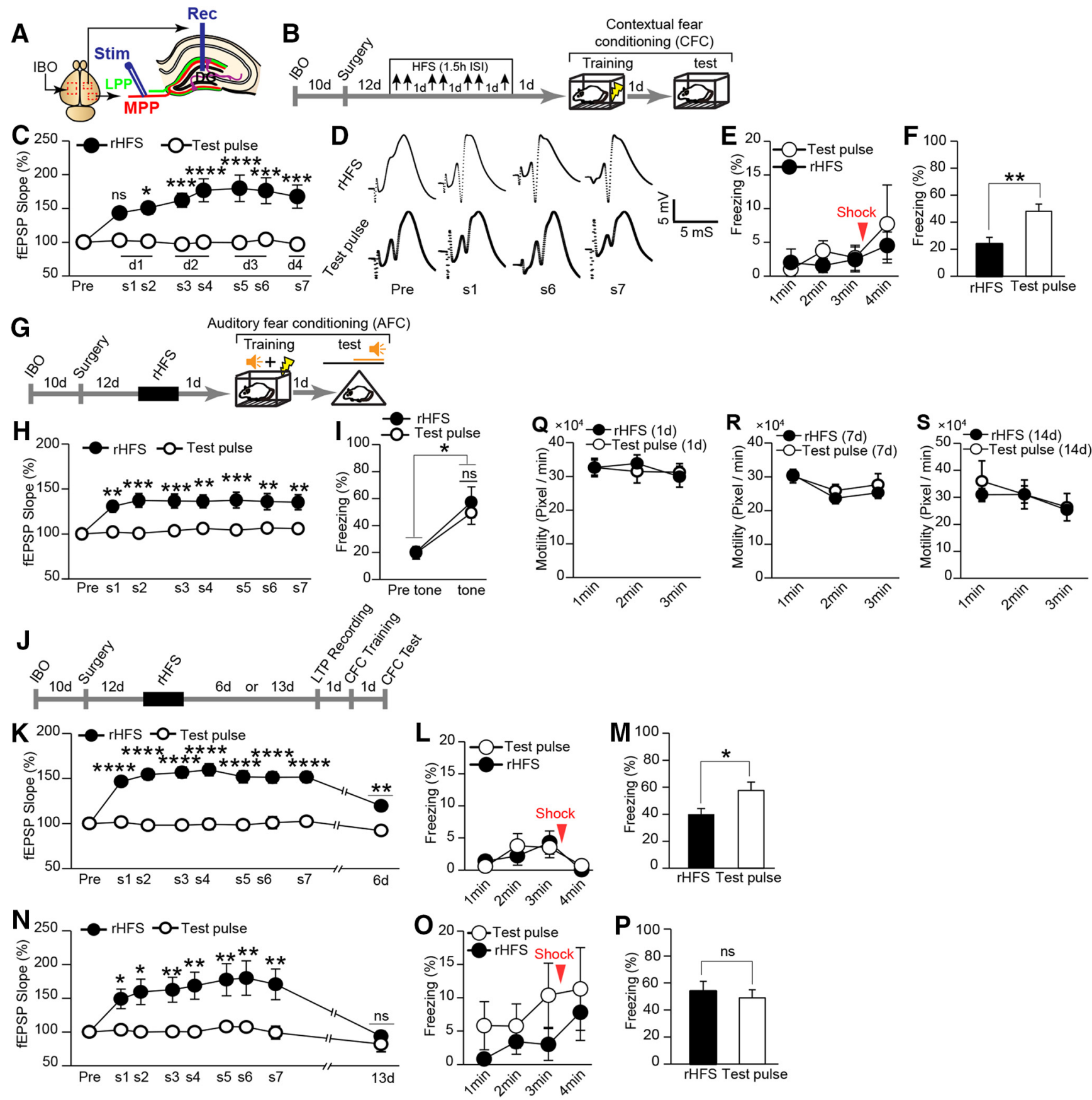

Figure 2. Recovery from hippocampal LTP saturation correlates with the memory capacity for CFC. A, Schematic illustration showing experimental setup for IBO injection and electrode implantation (for detailed information regarding the coordinates of the IB0 injection, see Table 1). MPP, Medial perforant path; LPP, lateral perforant path; Rec, recording electrode; Stim, stimulating electrode. $\boldsymbol{B}$, Experimental schedule for the effect of rHFS. Top arrows indicate the stimulation session. $\boldsymbol{C}$, Saturation of hippocampal LTP. The normalized values for the fEPSP slope for $r H F S$ ( $n=9$ ) and test pulse $(n=7)$ rats [(two-way ANOVA, $p=0.0009, F_{1,14}=17.58$, rHFS vs test pulse $(s 1, p=0.1 ; s 2, p=0.01 ; s 3, p=0.0008 ; s 4, p<0.0001 ; 55, p<0.0001 ; 56, p=0.0001 ; s 7, p=$ 0.0002), Bonferroni corrected for multiple comparisons)]. s, Session. $\boldsymbol{D}$, Example traces of evoked fEPSP responses. Pre, Before rHFS. $\boldsymbol{E}$, Freezing time during the training session (rHFS, $n=9$; test pulse, $n=7$ rats; two-way ANOVA, $p=0.5589, F_{1,14}=0.3584$ ). $\boldsymbol{F}$, Average freezing response during the test session ( $\mathrm{rHFS}, n=9$; test pulse, $n=7$ rats; unpaired $t$ test, $p=0.0051, t_{14}=3.31$ ). $\boldsymbol{G}$, Experimental schedule for AFC. $\boldsymbol{H}$, Normalized values for the fEPSP slope in rHFS and test pulse rats $\left(n=9\right.$ rats/group; two-way ANOVA, $p=0.001, F_{1,16}=16.11$, Bonferroni corrected for multiple comparisons). $I$, Freezing response before tone (pre) and during tone period (tone; $n=9$ rats $/$ group) in the AFC test (paired $t$ test, rHFS pre-tone vs tone, $p=0.01, t_{8}=3.18$; test pulse pre-tone vs tone, $p=0.01, t_{8}=3.19$; unpaired $t$ test, rHFS tone vs test pulse tone, $p=0.58, t_{16}=0.56$ ). J, Experimental schedule to check the gradual decay of synaptic efficacy and recovery of learning capacity. $\boldsymbol{K}, \mathrm{LTP}$ partially decayed after $6 \mathrm{~d}$ (two-way ANOVA, $p<0.0001, F_{1,15}=63.82 ; \mathrm{rHFS} \mathrm{d} 6$ v s test pulse $\mathrm{d} 6, p=0.0012$; Bonferroni corrected for multiple comparisons). $\boldsymbol{L}$, Freezing time during the training session (two-way ANOVA, $p=0.8422, F_{1,15}=0.0410$; test pulse, $n=9 ;$ rHFS, $n=8$ rats). $M$, Incomplete recovery of learning capacity (unpaired $t$ test, $p=0.03, t_{15}=2.29$ ) $7 \mathrm{~d}$ after the last HFS (test pulse, $n=9$; rHFS, $n=8$ rats). $N$, LTP returned to the baseline level $13 \mathrm{~d}$ after rHFS (two-way ANOVA, $p=0.0065, F_{1,14}=10.18$; rHFS d13 vs test pulse d13, $p>0.99 ;$ B Bonferroni corrected for multiple comparisons). $\boldsymbol{O}$, Freezing time during the training session (two-way ANOVA, $p=0.2778, F_{1,14}=1.275 ; n=8$ rats/group). $\boldsymbol{P}$, Recovery of learning capacity (unpaired $t$ test, $p=0.60, t_{14}=0.53, n=8$ rats/group). $\mathbf{Q}-\mathbf{S}$, Motility of the rHFS- and test pulse-treated rats on day 1 ( $\mathbf{Q}$, two-way ANOVA, $p=0.91, F_{1,14}=0.01 ;$ rHFS, $n=9 ;$ test pulse, $n=7$ rats), day 7 ( $\boldsymbol{R}$, two-way ANOVA, $F_{1,15}=0.37, p=0.55 ;$ rHFS, $n=8$; test pulse, $n=9$ rats), and day 14 (S, two-way ANOVA, $p=0.74, F_{1,14}=0.11 ; n=8$ rats/group) after the last HFS. ${ }^{*} p<0.05,{ }^{* *} p<$ $0.01,{ }^{* * *} p<0.001,{ }^{* * * *} p<0.0001$; ns, not significant. 


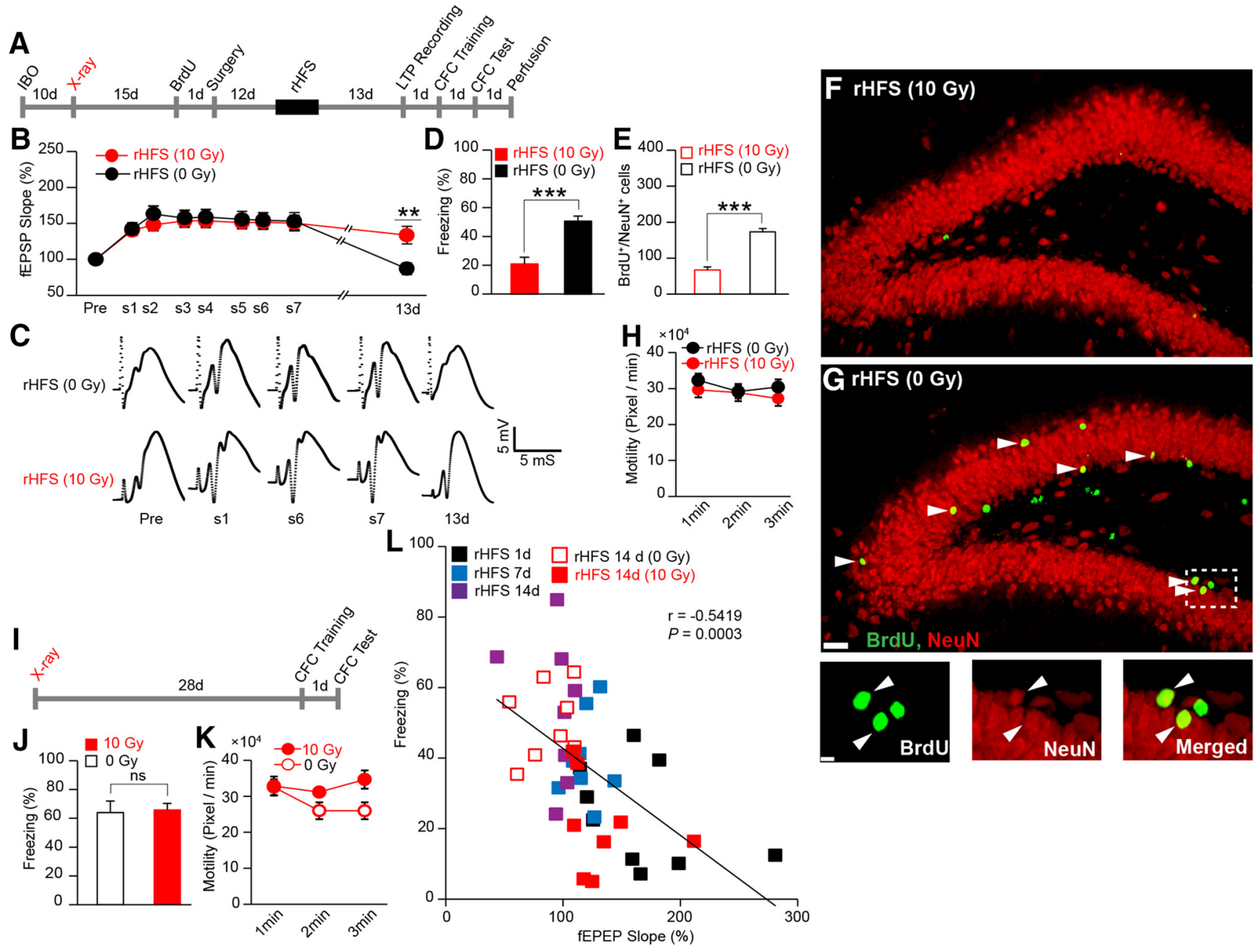

Figure 3. Adult hippocampal neurogenesis regulates the recovery from rHFS-induced impairment of CFC. $A$, Experimental schedule. $B$, LTP maintenance in nonirradiated ( 0 Gy) and $x$-rayirradiated (10 Gy) rats [two-way ANOVA, $p=0.91, F_{1,14}=0.01$; rHFS (10 Gy) $13 \mathrm{~d}$ vs rHFS ( 0 Gy) $13 \mathrm{~d}$, unpaired $t$ test, $p=0.006, t_{14}=3.23, n=8$ rats/group]. C, Example traces of evoked fEPSP responses. Pre, Before rHFS. D, Freezing response during the test session (unpaired $t$ test, $p=0.0002, t_{14}=4.87, n=8$ rats/group). $\boldsymbol{E}$, Reduced neurogenesis in the rHFS-irradiated rats ( $n=3$ rats/group, unpaired $t$ test, $\left.p=0.0009, t_{4}=8.77\right) . F, G$, Hippocampal coronal sections. Scale bar, $200 \mu \mathrm{m}$. Arrowheads indicate BrdU ${ }^{+} / \mathrm{NeuN}^{+}$double-positive cells. G, Bottom, magnified images from rHFS ( 0 Gy): BrdU (left), NeuN (middle), and merged (right). Scale bar, $50 \mu$ m. $\boldsymbol{H}$, Effect of rHFS and x-ray irradiation on the motility of the animals $14 \mathrm{~d}$ after the last HFS (two-way ANOVA, $p=$ $0.37, F_{115}=0.83 ; n=8$ rats/group). I, Experimental schedule for the effect of $\mathrm{x}$-ray on (FC. J, Freezing response at test (10 Gy, $n=8 ; 0 \mathrm{~Gy}, n=7$ rats; unpaired $t$ test, $p=0.84, t_{13}=0.19$ ). $\boldsymbol{K}$, Effect of $\mathrm{x}$-ray irradiation on the general activity of the animals $28 \mathrm{~d}$ after $\mathrm{x}$-ray irradiation (two-way ANOVA, $p=0.07, F_{1,13}=3.64 ; 10 \mathrm{~Gy}, n=8 ; 0 \mathrm{~Gy}, n=7$ rats). $L$, Correlation of $L$ TP and learning ability (Pearson correlation test, $r=-0.5419, p=0.0003$ ). Each dot represents data from an individual animal. ${ }^{*} p<0.05,{ }^{* *} p<0.01,{ }^{* * *} p<0.001 ; n s$, not significant.

day after the last HFS session, the animals were trained with the CFC task. There was no difference in freezing level during the conditioning session between the groups (Fig. 2E). The rHFS group showed lower freezing in the test session than the test pulse group $1 \mathrm{~d}$ after training (Fig. $2 F$ ). DG-LTP saturation had no effect on AFC, a hippocampus-independent learning task, because both the rHFS and test pulse groups showed the same freezing in the tone session $1 \mathrm{~d}$ after training (Fig. $2 G-I$ ). Therefore, saturation of hippocampal LTP impaired hippocampus-dependent memory without affecting hippocampus-independent memory.

LTP partially decayed from the saturation level $6 \mathrm{~d}$ after the last HFS session (Fig. $2 J, K$ ). The next day, animals were trained with the CFC task. There was no difference in freezing level during the training session (Fig. 2L). The rHFS group showed lower freezing than the test pulse group during the test session on the following day (Fig. 2J,M), but higher freezing than the rHFS group that performed CFC training $1 \mathrm{~d}$ after rHFS (unpaired $t$ test, $p=0.03, t_{15}=2.397$; Fig. $2 F$ ). LTP had completely decayed with a return to the baseline level $13 \mathrm{~d}$ after the last HFS (Fig.
$2 J, N)$. The following day, the animals were trained with the CFC task. Both the test pulse and rHFS groups showed comparable freezing in the test session $1 \mathrm{~d}$ after the training period without showing any difference in the training session (Fig. $2 J, O, P$ ). General activity during the training session of the CFC task was comparable between the rHFS and control groups (Fig. 2Q-S). Therefore, hippocampal memory capacity gradually recovered from saturation in parallel with the gradual decay of LTP.

Decreased hippocampal neurogenesis extends the recovery of memory capacity

We then assessed the role played by neurogenesis in the recovery of learning capacity. Rats were injected with IBO, and the whole brain was focally irradiated $10 \mathrm{~d}$ later, followed by LTP saturation and CFC training (Fig. $3 A$ ). rHFS in both the nonirradiated and irradiated groups saturated hippocampal LTP, with no significant between-group difference in the magnitude of LTP (Fig. $3 B, C)$. LTP in the nonirradiated group decayed and returned to the basal level $13 \mathrm{~d}$ after the last HFS. In contrast, the irradiated 

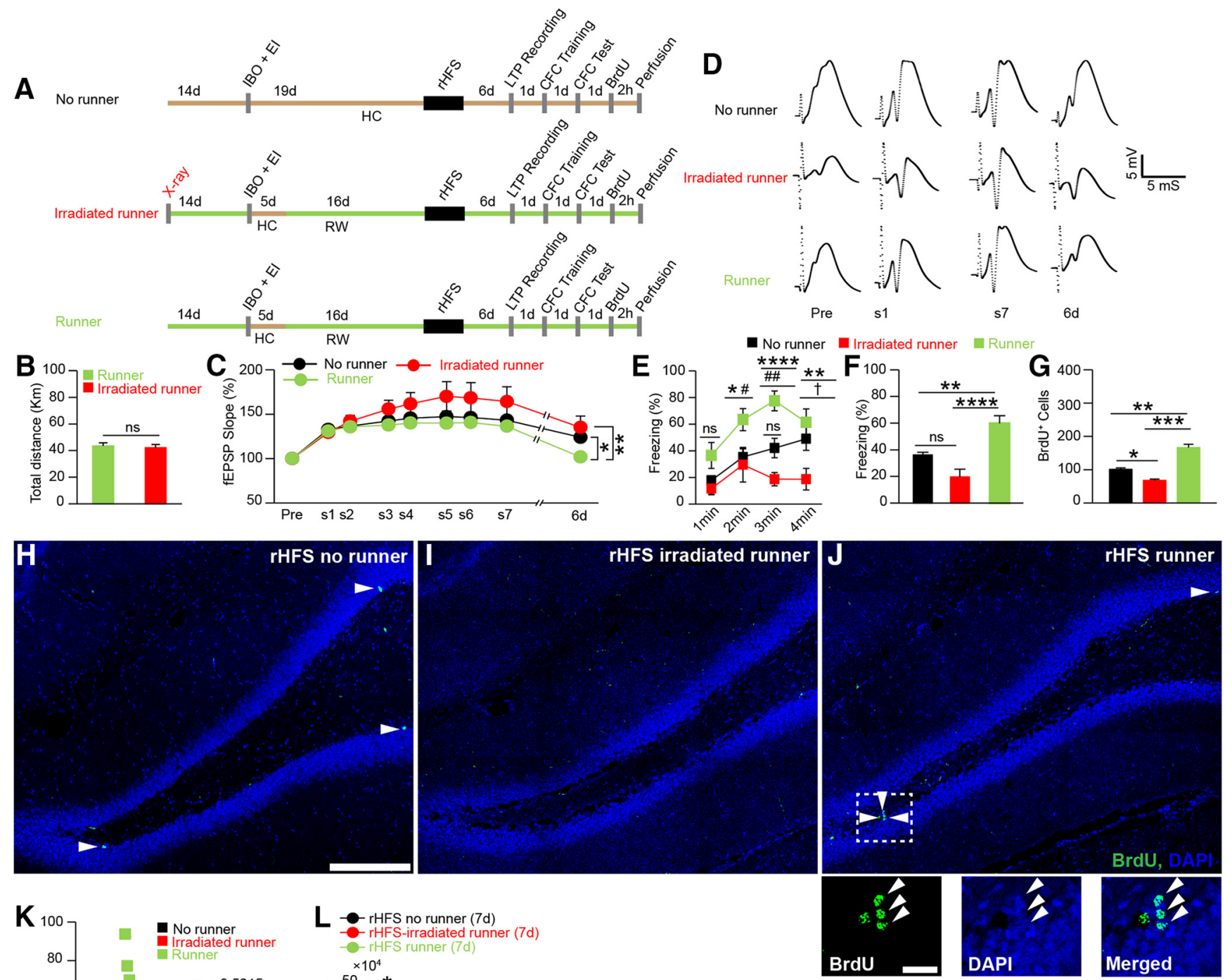

rHFS runner

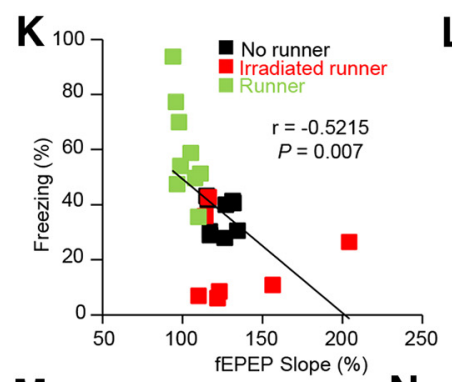

L - rHFS no runner (7d)
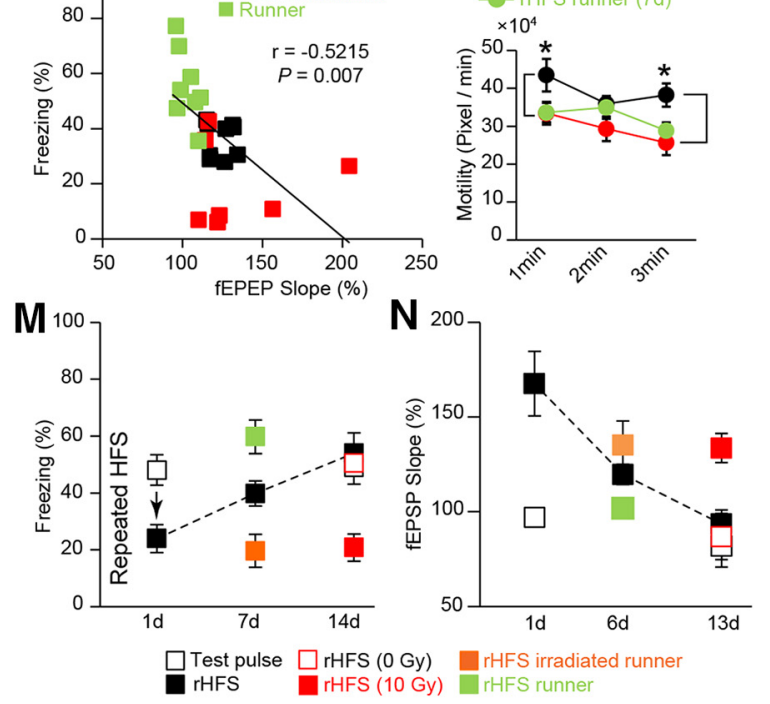

Figure 4. Adult hippocampal neurogenesis accelerates the recovery of rHFS-induced memory impairment. $A$, Experimental schedule. Green or ochre bars indicate housed with (running wheel, $\mathrm{RW}$ ) or without (HC) a running wheel, respectively. IBO $+\mathrm{El}, \mathrm{IBO}$ injection and electrode implantation. $\boldsymbol{B}$, Total distance traveled (runner, $n=9$; irradiated runner, $n=7$ rats; unpaired $t$ test, $p=$ $0.72, t_{14}=0.36$ ). C, LTP maintenance [two-way ANOVA, $p=0.07, F_{2,22}=2.89$ (one-way ANOVA, $p=0.005, F_{2,22}=6.54$, Fisher's LSD post hoc test, runner $6 \mathrm{~d}$ vs nonrunner $6 \mathrm{~d}, p=0.02 ;$ runner $6 \mathrm{~d}$ vs irradiated runner $6 \mathrm{~d}, p=0.002)$, nonrunner, $n=9$; runner, $n=9$; irradiated runner, $n=7$ rats]. $\boldsymbol{D}$, Example traces of evoked fEPSP responses. Pre, Before rHFS. $\boldsymbol{E}$, $\boldsymbol{F}$, Time courses of freezing $\left(\boldsymbol{E}\right.$, two-way ANOVA, $p<0.0001, F_{2,22}=17.04$, Tukey's multiple-comparisons test) and average freezing responses $\left(\boldsymbol{F}\right.$, one-way ANOVA, $p<0.0001, F_{2,22}=17.0$, Tukey's multiple-comparisons test) during CFC test (nonrunner, $n=9$; runner, $n=9$; irradiated runner, $n=7$ rats). G, Proliferation of the new neurons ( $n=3$ rats/group, one-way ANOVA, $p=0.0001, F_{2,6}=58.1$, Tukey's multiple-comparisons test). $\boldsymbol{H}-\boldsymbol{J}$, Hippocampal coronal sections from a nonrunner $(\boldsymbol{H})$, an irradiated runner $(\boldsymbol{I})$, and a runner rats $(\boldsymbol{J})$. Scale bar, $100 \mu \mathrm{m}$. Arrowheads indicate BrdU-positive cells. J, Bottom, Magnified images. Scale bar, $50 \mu \mathrm{m}$. BrdU (left), DAPI (middle), and merged (right) images. $\boldsymbol{K}$, Correlation of hippocampal LTP and learning ability (Pearson's correlation test, $r=$ $-0.5215, p=0.0075$ ). Each dot represents data from an individual animal. $L$, Effect of $x$-ray irradiation and running wheel on the general activity of the animals $6 \mathrm{~d}$ (Figure legend continues.) 


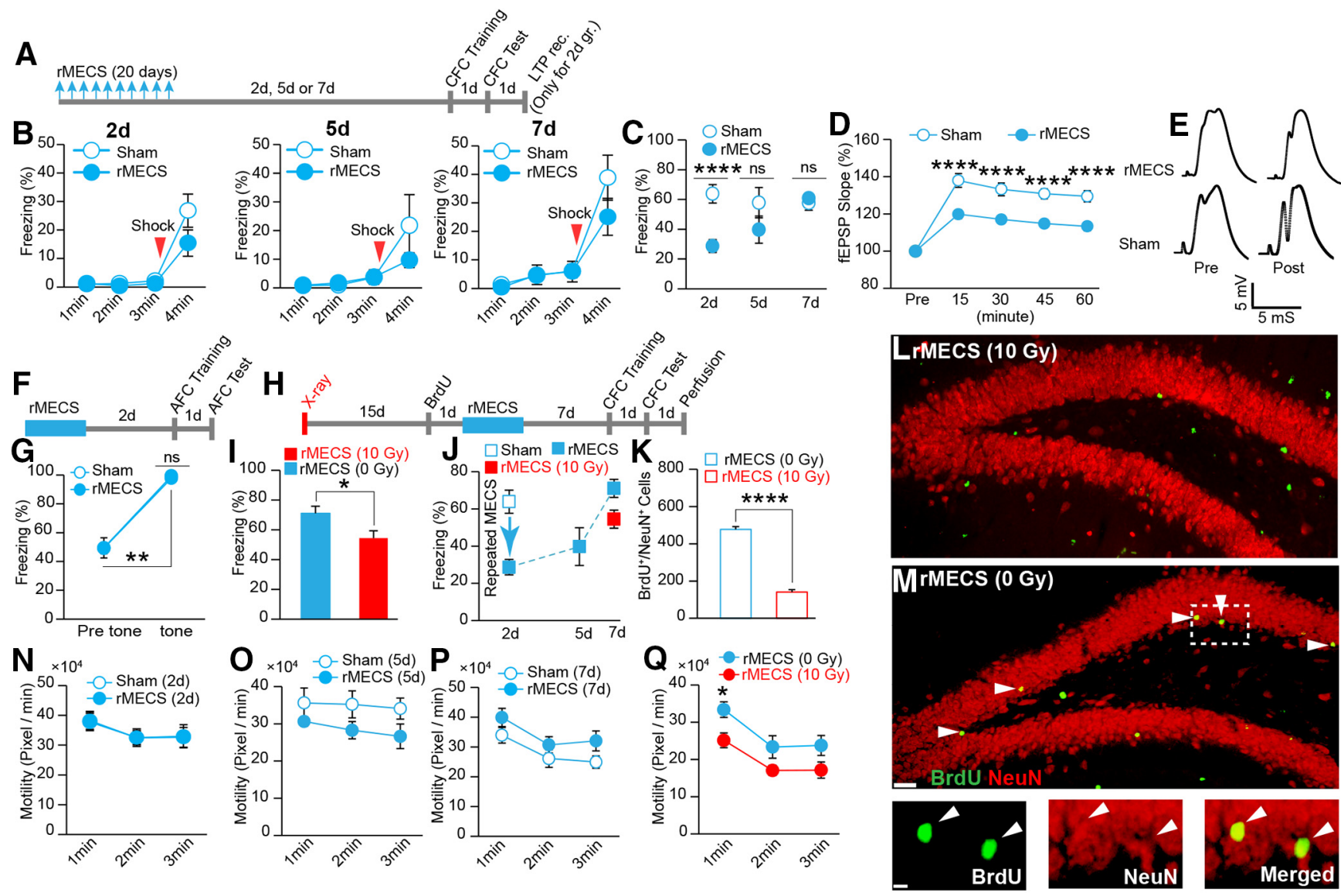

Figure 5. Adult hippocampal neurogenesis facilitates recovery from rMECS-induced impairment of CFC. $\boldsymbol{A}$, Experimental schedule. Top arrows indicate the MECS induction. $\boldsymbol{B}$, Freezing time during the training session. (left: rMECS-2d group, two-way ANOVA, $p=0.1200, F_{1,24}=2.599, n=13$ rats/group; middle: $r M E C S-5$ d group, two-way ANOVA, $p=0.4028, F_{1,11}=0.7571$, rMECS, $n=7$; sham, $n=6$ rats; right: rMECS-7d group, two-way ANOVA, $p=0.3691, F_{1,23}=0.8394 \mathrm{rMECS}, n=15$; sham, $n=10$ rats). C, Mean freezing response during the test. rMECS-2d groups (unpaired $t$ test, $p<0.0001, t_{24}=4.72, n=13$ rats/group), $5 \mathrm{~d}$ groups (unpaired $t$ test, $p=0.20, t_{11}=1.33$, rMECS, $n=7$; sham, $n=6$ rats), or $7 \mathrm{~d}$ groups (unpaired $t$ test, $p=0.58, t_{23}=$ 0.55, rMECS, $n=15$; sham, $n=10$ rats) after the last MECS. D, LTP induction in PP-DG synapses after CFC (two-way ANOVA, $p=0.0002, F_{1,15}=25.04$, Bonferroni corrected, sham, $n=7$; rMECS, $n=10$ rats). $\boldsymbol{E}$, Example traces of evoked fEPSP responses recorded before (pre) and after (post) tetanization. $\boldsymbol{F}$, Experimental schedule for AFC. $\boldsymbol{G}$, Averaged freezing response before tone (pre) and during (tone) tone period (Wilcoxon matched-pairs signed-rank test; rMECS pre-tone vs tone, $p=0.003$, sham pre-tone vs tone, $p=0.002 ;$ rMECS tone vs sham tone, Mann-Whitney test, $p=$ 0.19 , Mann-Whitney $U=29 ;$ sham, $n=10 ; \mathrm{rMECS}, n=9 \mathrm{rats}$ ). $\boldsymbol{H}$, Experimental schedule for CFC. $\boldsymbol{I}$, Freezing response during the (FC test (unpaired $t$ test, $p=0.02, t_{18}=2.41, n=10$ rats $/$ group). $J$, Summary of the behavioral results. $K$, Number of BrdU ${ }^{+} / \mathrm{NeuN}^{+}$double-positive cells $\left(n=4\right.$ rats/group, unpaired $t$ test, $p<0.0001, t_{6}=16.99$ ). $L, M$, Hippocampal coronal section from rMECS-irradiated rats $(\boldsymbol{L})$ and rMECS-nonirradiated rats $(\boldsymbol{M})$. Scale bar, $200 \mu \mathrm{m}$. Arrowheads indicate BrdU ${ }^{+} / \mathrm{NeuN}^{+}$double-positive cells. Magnified images from an $\mathrm{rMECS}$-nonirradiated rat (M; bottom); BrdU (left), NeuN (middle), and merged (right). Scale bar, $50 \mu \mathrm{m} . \boldsymbol{N}-\boldsymbol{Q}$, Motility of the sham- or rMECS-treated animals on $2 \mathrm{~d}\left(\boldsymbol{N}\right.$, two-way ANOVA, $p=0.99, F_{1,24}=5.6 \mathrm{e}-006 ; n=$ 13 rats $/$ group $), 5 \mathrm{~d}\left(\boldsymbol{0}\right.$, two-way ANOVA, $p=0.09, F_{1,11}=3.40, \mathrm{rMECS}, n=7$; sham, $\left.n=6\right)$, and $7 \mathrm{~d}\left(\boldsymbol{P}\right.$, two-way ANOVA, $p=0.09, F_{1,23}=2.96, \mathrm{rMECS}, n=15 ;$; sham, $n=10$ ) from the last MECS . Q, Effect of rMECS and $x$-ray irradiation on the motility of the animals during their training session $7 \mathrm{~d}$ after the last MECS (two-way ANOVA, $p=0.0067, F_{1,18}=9.37$, rMECS irradiated vs rMECS nonirradiated, $1 \mathrm{~min}, p=0.03$, Bonferroni corrected, $n=10$ rats/group). ${ }^{*} p<0.05,{ }^{* *} p<0.01,{ }^{* * *} p<0.001,{ }^{* * * *} p<0.0001$; ns, not significant.

group retained LTP for $13 \mathrm{~d}$. On the following day, the animals were trained with the CFC task. The irradiated group showed significantly lower freezing in test sessions $1 \mathrm{~d}$ after training than the nonirradiated group (Fig. 3D) and also had significantly lower neurogenesis than the nonirradiated group (Fig. $3 E-G$ ). Both groups showed comparable motility in the training session (Fig. $3 H$ ). X-ray irradiation alone had no effect on CFC learning ability because irradiated rats that did not receive rHFS stimulation showed freezing in the test session $1 \mathrm{~d}$ after CFC training that

$\leftarrow$

(Figure legend continued.) after the last HFS induction (two-way ANOVA, $p=0.01, F_{2,22}=$ 5.26 , rHFS nonrunner vs rHFS runner, $1 \mathrm{~min}, p=0.046$, rHFS nonrunner vs rHFS-irradiated runner, $3 \mathrm{~min}, p=0.014$, Tukey's multiple-comparisons test; rHFS nonrunner, $n=9$; rHFS runner, $n=9$; rHFS-irradiated runner, $n=7$ rats). $\boldsymbol{M}, \boldsymbol{N}$, Summary of the behavioral $(\boldsymbol{M})$ and LTP $(\boldsymbol{N})$ results from Figures 2,3 , and $4 .{ }^{*} p<0.05,{ }^{* *} p<0.01,{ }^{* * *} p<0.001,{ }^{* * *} p<$ 0.0001 , ns, not significant, $\# p=0.03$, \#\# =0.004 (runner vs nonrunner), $t p=0.02$ (nonrunner vs irradiated runner). was comparable to that shown by the nonirradiated rats (Fig. $3 I, J)$. Motility during the training session was not affected by irradiation (Fig. $3 \mathrm{~K}$ ). There was a significant negative correlation between the fEPSP slope and freezing (Fig. 3L). Therefore, reduced neurogenesis postponed the recovery of hippocampusdependent learning capacity by maintaining saturation of hippocampal LTP.

Increased hippocampal neurogenesis speeds up the recovery of memory capacity

The voluntary running wheel exercise (van Praag et al., 1999; Kitamura et al., 2009) was used to examine the effect of increased neurogenesis on recovery from LTP saturation (Fig. 4). One day after irradiation, rats were housed in running wheel cages for the entire remaining session except for the $5 \mathrm{~d}$ immediately after the IBO injection (Fig. 4A). The distance run was comparable between the nonirradiated and irradiated rats (Fig. $4 B$ ). One month after the onset of the exercise regimen, rats were subjected to 
rHFS. There was no between-group difference in the fEPSP slope during rHFS (Fig. 4C,D). Six days after rHFS, the runner rats showed faster decay of LTP saturation than the nonrunner and irradiated runner groups (Fig. 4C). The next day, the animals were subjected to CFC training. The runner group showed complete recovery of memory capacity, whereas the other groups showed impaired memory capacity in the test session $1 \mathrm{~d}$ after the training (Fig. 4E, F; see also Fig. 2M). Rats were administered BrdU $1 \mathrm{~d}$ after the CFC test, and $2 \mathrm{~h}$ later they were perfused and analyzed for cell proliferation. The 6-week running exercise resulted in more cell proliferation in the subgranular zone of the DG in the runner group than in the nonrunner and irradiated runner groups (Fig. $4 G-J$ ). Data from individual animals showed a significant negative correlation between fEPSP slope and freezing (Fig. $4 K$ ). Motility during the training session was comparable between the rHFS-irradiated runner group and the rHFS nonirradiated runner group (Fig. $4 L$ ). Together, these results show that ablation and increased neurogenesis delayed and accelerated LTP decay, respectively, and that accelerated LTP decay was accompanied by slow or rapid recovery of memory capacity (Fig. $4 M, N$ ).

\section{Adult hippocampal neurogenesis assists the recovery from rMECS-induced memory impairment}

Whole-brain stimulation with rMECS is another way to saturate hippocampal synaptic efficacy (Stewart et al., 1994). Rats received 10 MECS and were then trained with the CFC task (Fig. 5A). The rMECS-2d group, in which the interval between the last MECS and CFC training was $2 \mathrm{~d}$, showed lower freezing than the sham group in the CFC test session $1 \mathrm{~d}$ after the training without showing a significant difference in the training session (Fig. $5 B$, left, $C$ ). LTP induced after CFC training was more impaired in the rMECS group than in the sham group (Fig. $5 D, E$ ). rMECS had no effect on the hippocampus-independent memory task (Fig. 5F,G). Therefore, as in the case of rHFS, rMECS saturated hippocampal LTP and impaired hippocampus-dependent memory capacity.

The rMECS-7d group showed comparable freezing to that of the sham group in the CFC test session $1 \mathrm{~d}$ after training (Fig. $5 C$ ). There was no difference in the freezing time during the training session between the groups (Fig. 5B, right). Therefore, an interval of $7 \mathrm{~d}$ was sufficient to recover completely memory capacity in the rMECS group.

The effect of irradiation was assessed by subjecting the animals to the same course of rMECS $18 \mathrm{~d}$ after irradiation (Fig. $5 \mathrm{H}$ ). Animals were trained with the CFC task $7 \mathrm{~d}$ after the last MECS. In the test session $1 \mathrm{~d}$ after CFC training, the $\mathrm{x}$-ray-irradiated group (rMECS + $10 \mathrm{~Gy}$ ) showed lower freezing than the nonirradiated group (rMECS + 0 Gy; Fig. $5 H-J$ ). The irradiated group also showed lower neurogenesis than the nonirradiated group (Fig. $5 K-M$ ). The motility of the animals during the training session of the CFC task was comparable between the rMECS groups and sham groups (Fig. $5 \mathrm{~N}-\mathrm{Q}$ ), indicating that rMECS had no effect on the general activity of the animals.

\section{Discussion}

Here, we demonstrate that rHFS and rMECS impair the hippocampusdependent memory without affecting hippocampus-independent memory. We did not observe a significant difference in the freezing time after the shock presentation between the groups, although there was a tendency of lower freezing in the rHFS- and rMECS-treated groups than the control animals. Hippocampus-dependent learning induces LTP in the hippocampus (Whitlock et al., 2006) and activity-dependent synaptic plasticity, LTP, and LTD at appropriate synapses are sufficient for information storage (Nabavi et al., 2014). Therefore, the artificially induced hippocampal LTP saturation may impair the acquisition and/or formation of fear memory.

Previous studies using the NMDA receptor antagonist AP5 (Morris et al., 1986) and mutant mice defective in $\alpha$-CaMKII (Silva et al., 1992) demonstrated that the blockade of hippocampal LTP impairs spatial learning in the Morris water maze task. Together with several other studies (Castro et al., 1989; Stewart et al., 1994; Moser et al., 1998), our results reestablish the predicted impairment of learning and/or memory after LTP saturation.

Moreover, this study suggests that the homeostatic plasticity required to remove old memories and make room for new ones is facilitated by adult neurogenesis. Our results are consistent with the neurocomputational model (Aimone et al., 2009; Weisz and Argibay, 2009), which purports that neurogenesis increases network capacity for new information while facilitating the removal of old information. Hippocampal neurogenesis slowly renormalizes the increase in synaptic strength resulting from LTP induced by strong tetanic stimulation, with the return to the basal level usually taking $\sim 2$ weeks. Therefore, sleep (Tononi and Cirelli, 2014; Li et al., 2017; de Vivo et al., 2017) and neurogenesis may play complementary roles by mediating rapid and slow scaling down of synaptic strength, respectively. Because hippocampal neurogenesis specifically renormalizes strong tetanus-induced long-lasting LTP, it may result in the decay of consolidated memories that escape sleep-mediated erasure from the hippocampus. This neurogenesis-mediated decay of hippocampal memory does not result in the loss of a memory because this process is accompanied by an increase in the neocortex dependence of the memory (Kitamura et al., 2009).

Synaptic competition between old and new neurons occurs when new neurons are integrated into preexisting circuits (Lledo et al., 2006; Toni et al., 2007; Zhao et al., 2008). Integration of new neurons has an impact on activity-dependent synaptic rewiring in the hippocampus (Toni et al., 2007; Ohkawa et al., 2012). Therefore, a blockade of new synapse integration stabilizes the hippocampal network, which then prevents the clearance of memories from the hippocampus. The replacement of synapses in this manner may maintain learning capacity because the naive synaptic connections made by the new neurons are flexible and can therefore be integrated into various networks that store newly acquired memories.

The level of adult hippocampal neurogenesis is regulated by many factors (Gould et al., 1999; Zhao et al., 2008; Deng et al., 2010; Ohkawa et al., 2012; Kitamura and Inokuchi, 2014), including neuronal activity, stress, and aging. A decrease in neurogenesis with aging is associated with an age-related decline in some forms of hippocampus-dependent memories (van Praag et al., 2005; Villeda et al., 2011). Therefore, reduced neurogenesis leading to a decrease in the clearance of old memories already stored in cortical networks may be the critical factor underlying the limited capacity of the hippocampus to acquire and store new information in aged animals.

\section{References}

Aimone JB, Wiles J, Gage FH (2009) Computational influence of adult neurogenesis on memory encoding. Neuron 61:187-202. CrossRef Medline

Altman J, Das GD (1965) Autoradiographic and histological evidence of postnatal hippocampal neurogenesis in rats. J Comp Neurol 124:319335. CrossRef Medline

Bernier BE, Lacagnina AF, Ayoub A, Shue F, Zemelman BV, Krasne FB, Drew MR (2017) Dentate gyrus contributes to retrieval as well as encoding: evidence from context fear conditioning, recall, and extinction. J Neurosci 37:6359-6371. CrossRef Medline 
Buzsáki G, Moser EI (2013) Memory, navigation and theta rhythm in the hippocampal-entorhinal system. Nat Neurosci 16:130-138. CrossRef Medline

Castro CA, Silbert LH, McNaughton BL, Barnes CA (1989) Recovery of spatial learning deficits after decay of electrically induced synaptic enhancement in the hippocampus. Nature 342:545-548. CrossRef Medline

Collingridge GL, Peineau S, Howland JG, Wang YT (2010) Long-term depression in the CNS. Nat Rev Neurosci 11:459-473. CrossRef Medline

Conway MA (2009) Episodic memories. Neuropsychologia 47:2305-2313. CrossRef Medline

Deitch AD, Moses MJ (1957) The nissl substance of living and fixed spinal ganglion cells. II. An ultraviolet absorption study. J Biophys Biochem Cytol 3:449-456. CrossRef Medline

Deng W, Aimone JB, Gage FH (2010) New neurons and new memories: how does adult hippocampal neurogenesis affect learning and memory? Nat Rev Neurosci 11:339-350. CrossRef Medline

de Vivo L, Bellesi M, Marshall W, Bushong EA, Ellisman MH, Tononi G, Cirelli C (2017) Ultrastructural evidence for synaptic scaling across the wake/sleep cycle. Science 355:507-510. CrossRef Medline

Fanselow MS (2010) From contextual fear to a dynamic view of memory systems. Trends Cogn Sci 14:7-15. CrossRef Medline

Fukazawa Y, Saitoh Y, Ozawa F, Ohta Y, Mizuno K, Inokuchi K (2003) Hippocampal LTP is accompanied by enhanced F-actin content within the dendritic spine that is essential for late LTP maintenance in vivo. Neuron 38:447-460. CrossRef Medline

Gould E, Beylin A, Tanapat P, Reeves A, Shors TJ (1999) Learning enhances adult neurogenesis in the hippocampal formation. Nat Neurosci 2:260265. CrossRef Medline

Hebb DO (1949) The organization of behavior. New York, NY: Wiley.

Humason GL (1983) Animal tissue techniques. San Francisco: W.H. Freeman.

Inokuchi K, Kato A, Hiraia K, Hishinuma F, Inoue M, Ozawa F (1996) Increase in activin beta A mRNA in rat hippocampus during long-term potentiation. FEBS Lett 382:48-52. CrossRef Medline

Jarrard LE (1989) On the use of ibotenic acid to lesion selectively different components of the hippocampal formation. J Neurosci Methods 29:251259. Medline

Kitamura T, Inokuchi K (2014) Role of adult neurogenesis in hippocampalcortical memory consolidation. Mol Brain 7:13. CrossRef Medline

Kitamura T, Saitoh Y, Takashima N, Murayama A, Niibori Y, Ageta H, Sekiguchi M, Sugiyama H, Inokuchi K (2009) Adult neurogenesis modulates the hippocampus-dependent period of associative fear memory. Cell 139:814-827. CrossRef Medline

Kitamura T, Okubo-Suzuki R, Takashima N, Murayama A, Hino T, Nishizono H, Kida S, Inokuchi K (2012) Hippocampal function is not required for the precision of remote place memory. Mol Brain 5:5. CrossRef Medline

Li W, Ma L, Yang G, Gan WB (2017) REM sleep selectively prunes and maintains new synapses in development and learning. Nat Neurosci 20: 427-437. CrossRef Medline

Liu X, Ramirez S, Pang PT, Puryear CB, Govindarajan A, Deisseroth K, Tonegawa S (2012) Optogenetic stimulation of a hippocampal engram activates fear memory recall. Nature 484:381-385. CrossRef Medline

Lledo PM, Alonso M, Grubb MS (2006) Adult neurogenesis and functional plasticity in neuronal circuits. Nat Rev Neurosci 7:179-193. CrossRef Medline

Matsuo R, Murayama A, Saitoh Y, Sakaki Y, Inokuchi K (2000) Identification and cataloging of genes induced by long-lasting long-term potentiation in awake rats. J Neurochem 74:2239-2249. Medline

Morris RG, Anderson E, Lynch GS, Baudry M (1986) Selective impairment of learning and blockade of long-term potentiation by an N-methyl-Daspartate receptor antagonist, AP5. Nature 319:774-776. CrossRef Medline

Moser EI, Krobert KA, Moser MB, Morris RG (1998) Impaired spatial learning after saturation of long-term potentiation. Science 281:2038 2042. CrossRef Medline

Nabavi S, Fox R, Proulx CD, Lin JY, Tsien RY, Malinow R (2014) Engineering a memory with LTD and LTP. Nature 511:348 -352. CrossRef Medline

Ofen N, Kao YC, Sokol-Hessner P, Kim H, Whitfield-Gabrieli S, Gabrieli JD (2007) Development of the declarative memory system in the human brain. Nat Neurosci 10:1198-1205. CrossRef Medline

Ohkawa N, Saitoh Y, Tokunaga E, Nihonmatsu I, Ozawa F, Murayama A, Shibata F, Kitamura T, Inokuchi K (2012) Spine formation pattern of adult-born neurons is differentially modulated by the induction timing and location of hippocampal plasticity. PLoS One 7:e45270. CrossRef Medline

Scoville WB, Milner B (1957) Loss of recent memory after bilateral hippocampal lesions. J Neurol Neurosurg Psychiatry 20:11-21. CrossRef Medline

Silva AJ, Paylor R, Wehner JM, Tonegawa S (1992) Impaired spatial learning in alpha-calcium-calmodulin kinase II mutant mice. Science 257: 206-211. CrossRef Medline

Stewart C, Jeffery K, Reid I (1994) LTP-like synaptic efficacy changes following electroconvulsive stimulation. Neuroreport 5:1041-1044. CrossRef Medline

Toni N, Teng EM, Bushong EA, Aimone JB, Zhao C, Consiglio A, van Praag H, Martone ME, Ellisman MH, Gage FH (2007) Synapse formation on neurons born in the adult hippocampus. Nat Neurosci 10:727-734. CrossRef Medline

Tononi G, Cirelli C (2014) Sleep and the price of plasticity: from synaptic and cellular homeostasis to memory consolidation and integration. Neuron 81:12-34. CrossRef Medline

van Praag H, Kempermann G, Gage FH (1999) Running increases cell proliferation and neurogenesis in the adult mouse dentate gyrus. Nat Neurosci 2:266-270. CrossRef Medline

van Praag H, Shubert T, Zhao C, Gage FH (2005) Exercise enhances learning and hippocampal neurogenesis in aged mice. J Neurosci 25:86808685. CrossRef Medline

Villeda SA, et al. (2011) The ageing systemic milieu negatively regulates neurogenesis and cognitive function. Nature 477:90-94. CrossRef Medline

Weisz VI, Argibay PF (2009) A putative role for neurogenesis in neurocomputational terms: inferences from a hippocampal model. Cognition 112:229-240. CrossRef Medline

Whitlock JR, Heynen AJ, Shuler MG, Bear MF (2006) Learning induces long-term potentiation in the hippocampus. Science 313:1093-1097. CrossRef Medline

Zhao C, Deng W, Gage FH (2008) Mechanisms and functional implications of adult neurogenesis. Cell 132:645-660. CrossRef Medline 